\title{
Modèle dynamique de la ligne poussoir de directions assistées électriques
}

\author{
François Besson ${ }^{1}$, Guy Ferraris ${ }^{2}$, Michèle Guingand ${ }^{2, a}$ et \\ JeAn-Pierre de Vaujany ${ }^{2}$ \\ 1 JTEKT Europe, ZI Le Brotteau, 69540 Irigny, France \\ 2 Université de Lyon, LaMCoS, INSA-Lyon, CNRS UMR 5259, 69621 Villeurbanne Cedex, France
}

Reçu le 10 juillet 2008, accepté le 21 janvier 2010

\begin{abstract}
Résumé - La dernière décennie a vu apparaître de nombreuses solutions technologiques destinées à améliorer le confort des automobilistes. L'une d'entre elles, la direction assistée électrique (DAE), a considérablement évolué : de plus en plus de véhicules en sont équipés. L'assistance, délivrée par l'intermédiaire d'un moteur électrique et d'un réducteur roue et vis sans fin, est contrôlée par un calculateur. Cette solution offre des possibilités plus intéressantes en terme de consommation d'énergie que les systèmes hydrauliques encore utilisés sur certains véhicules. La compréhension et la caractérisation du comportement dynamique des DAE sont devenues des préoccupations majeures. En effet, afin de répondre à des exigences vibratoires et acoustiques toujours plus sévères, le comportement sonore et vibratoire de la direction est étudié. Dans ce contexte, le comportement dynamique de l'ensemble de la DAE est simulé. L'accent a notamment été mis sur la modélisation des deux engrenages présents dans le système (roue-vis sans fin et pignon-crémaillère) et leurs systèmes à rattrapage de jeu. La modélisation de la ligne poussoir des DAE est présentée, ainsi que sa validation et son intégration dans un modèle dynamique d'ensemble. Cette étude consiste en une approche à la fois numérique et expérimentale de la problématique étudiée.
\end{abstract}

Mots clés : Direction assistée / dynamique / non linéaire / réponse transitoire / engrenage pignon-crémaillère / ligne poussoir

\begin{abstract}
Dynamic model of the yoke line of EPS. During the last decade, many new technical solutions dedicated to the driver's comfort have raised. One of these solutions was the Power Steering which is still evolving much: more and more vehicles are equipped with electrical power steering (EPS). This kind of steering permits better performances in terms of energy consumption than hydraulic systems, still in use on some vehicles. The assistance torque is delivered by an electrical motor controlled by a special unit. This torque is applied through a worm gear to the shaft. The comfort in terms of wheel vibration and noise in the passenger compartment induced by the EPS makes the understanding and characterization of the dynamic behavior one of the leading for EPS development. In this context was started a study which aims to go further into global dynamic behavior of EPS. A model was built up where the stress was put on the gears models (worm gear and rack-and-pinion) and their anti-backlash systems (ABLS for the worm gear and yoke line for the rack-and-pinion). The modelling of the yoke line is developed, and also its validation and its integration in a global model of an EPS. The study was also experimental in order to correlate the simulation results.
\end{abstract}

Key words: EPS / dynamic / non linear / transient response / rack-and-pinion gear / yoke line

a Auteur pour correspondance : michele.guingand@insa-lyon.fr 


\section{Nomenclature}

\begin{tabular}{|c|c|c|}
\hline$a$ & Décalage vertical des centres de courbure & $\mathrm{m}$ \\
\hline ABLS & Anti BackLash System & \\
\hline$b$ & Décalage horizontal des centres de courbures & $\mathrm{m}$ \\
\hline$c$ & Élément d'amortissement & N.m ${ }^{-1} \cdot \mathrm{s}$ \\
\hline$C$ & Amortissement & N.m ${ }^{-1} \cdot \mathrm{s}$ \\
\hline$d=\sqrt{a^{2}+b^{2}}$ & Décalage des centres de courbure & $\mathrm{m}$ \\
\hline $\mathrm{D}$ & Centre de courbure du poussoir & \\
\hline DAE & Direction Assistée Électrique & \\
\hline ddl & Degré de liberté & \\
\hline DM & Direction Manuelle & \\
\hline$f$ & Fonction jeu & \\
\hline$F$ & Effort & $\mathrm{N}$ \\
\hline$H$ & Hauteur du poussoir & $\mathrm{m}$ \\
\hline$I$ & Inertie & kg. $\mathrm{m}^{2}$ \\
\hline$k$ & Élément de raideur & N.m ${ }^{-1}$ \\
\hline$K$ & Raideur & N.m ${ }^{-1}$ \\
\hline$L$ & Longueur & $\mathrm{m}$ \\
\hline$m$ & Élément de masse & $\mathrm{kg}$ \\
\hline$M$ & Masse & $\mathrm{kg}$ \\
\hline$\vec{n}$ & Normale au contact & \\
\hline$q$ & Déplacements aux nœuds d'une structure & $\mathrm{m}$ \\
\hline$R$ & Énergie de dissipation & $\mathrm{J}$ \\
\hline$R(X, Y, Z)$ & Trièdre droit & \\
\hline$t$ & Temps & $\mathrm{s}$ \\
\hline$T$ & Énergie cinétique & $\mathrm{J}$ \\
\hline$U$ & Énergie de déformation & $\mathrm{J}$ \\
\hline$v$ & Déplacement sur $Y$ & $\mathrm{~m}$ \\
\hline$w$ & Déplacement sur $Z$ & $\mathrm{~m}$ \\
\hline$\alpha$ & Angle de contact & $\mathrm{rad}$ \\
\hline$\delta$ & Configuration des éléments non-linéaires & \\
\hline$\Delta$ & Écrasement & $\mathrm{m}$ \\
\hline$\theta$ & Rotation autour de $X$ & $\mathrm{rad}$ \\
\hline$c_{\alpha} \equiv \cos \alpha$ & $s_{\alpha} \equiv \sin \alpha$ & \\
\hline[]$/\{\}$ & Matrice/vecteur & \\
\hline$\dot{x}$ & Dérivée temporelle première de $x$ (vitesse) & \\
\hline$\ddot{x}$ & Dérivée temporelle seconde de $x$ (accélération) & \\
\hline c & Indice de contact & \\
\hline $\mathrm{G}$ & Indice relatif au centre de gravité du poussoir & \\
\hline $\mathrm{h}$ & Indice horizontal & \\
\hline$i$ & Indice d'incrémentation & \\
\hline $\mathrm{O}$ & Indice relatif au centre de la crémaillère & \\
\hline $\mathrm{p}$ & Indice relatif au poussoir & \\
\hline$v$ & Indice vertical & \\
\hline
\end{tabular}

\section{Introduction}

\subsection{Les directions assistées électriques}

Une direction automobile est composée de plusieurs carters et d'une chaîne cinématique qui relie le volant aux extrémités des biellettes, figure 1. Lors de la rotation du volant, la crémaillère ainsi que les biellettes sont entraînées en translation par l'intermédiaire de la colonne de direction et de l'engrenage pignon-crémaillère. Les biellettes étant reliées aux roues par l'intermédiaire du porte fusée, leur translation provoque le pivotement des roues.

Il existe plusieurs types de DAE, notamment les directions assistées sur colonne (C-EPS : Column-Electric Power Steering), sur crémaillère (R-EPS : Rack-Electric
Power Steering) et sur pignon (P-EPS : Pinion-Electric Power Steering). L'étude exposée ici ne porte que sur ces dernières. La chaîne cinématique d'un système P-EPS comprend 3 blocs : la direction manuelle, le module d'assistance et la colonne de direction, non représentée sur la figure 1 .

\subsubsection{Direction manuelle}

La direction manuelle d'une DAE, figure 2, est similaire aux directions non assistées. Elle comporte l'engrenage pignon-crémaillère, un système à rattrapage de jeu (ligne poussoir), un palier et les biellettes. Ces dernières ne sont pas incluses dans l'étude. 


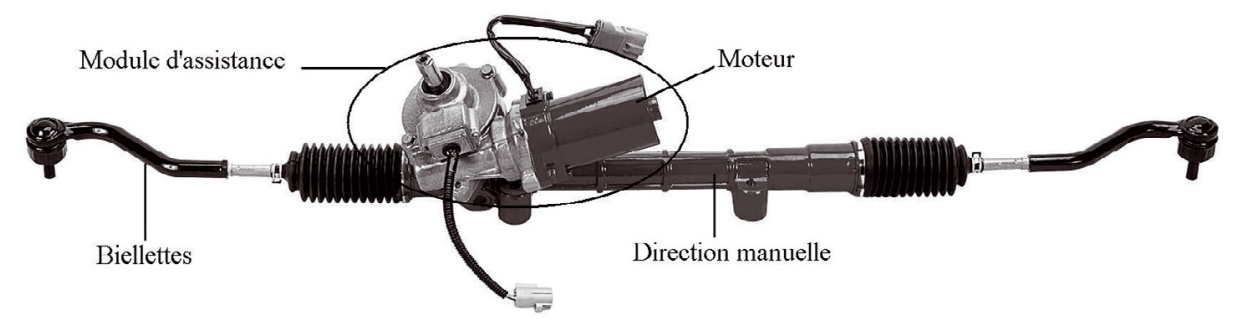

Fig. 1. Vue d'ensemble d'une P-EPS.

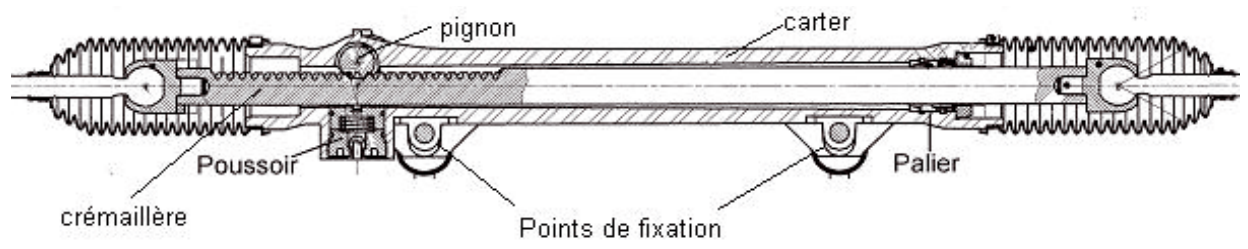

Fig. 2. Direction manuelle.

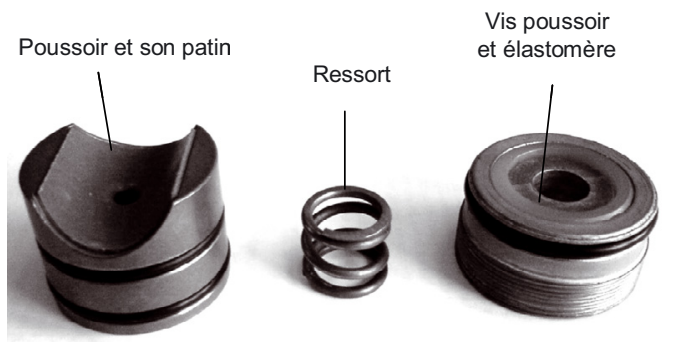

Fig. 3. Pièces constitutives de la ligne poussoir.

La ligne poussoir, figure 3, est composée du poussoir et de son patin, d'une vis poussoir (liée au carter de la direction) dans laquelle est fichée une bague élastomère et d'un ressort entre ces deux pièces. Son comportement définit les mouvements radiaux possibles pour le crémaillère et donc pour les biellettes, directement liées au véhicule.

\subsubsection{Module d'assistance}

L'assistance au conducteur, transmise par un engrenage roue et vis sans fin, figure 4, est assurée grâce à un moteur électrique lié à la vis. Un système à rattrapage de jeu (ressort ABLS) peut être associé à cet engrenage.

La chaîne cinématique du module d'assistance est également composée de trois arbres : le pignon, la barre de torsion et l'arbre d'entrée. Le couple d'assistance, transmis par l'engrenage roue et vis sans fin au pignon (grand rapport de réduction), est déterminé par le calculateur à partir de la mesure de la déformation de la barre de torsion et de la vitesse du véhicule.

\subsubsection{Colonne de direction}

La colonne est composée de deux arbres, de roulements et d'une transmission à double cardan, figure 5. Elle est fixée sur la traverse du véhicule par l'intermédiaire d'un carter.

\subsection{Les vibrations dans une DAE}

Les principales sources de vibrations identifiées dans les DAE sont les deux engrenages et leurs systèmes à rattrapage de jeu. Considérons, par exemple, le sous-ensemble pignon-crémaillère-ligne poussoir. Le jeu d'engrènement du pignon-crémaillère peut être considéré comme nul, car très faible. L'engrenage est alors en configuration 3, figure 6 . À chaque montée en couple, la crémaillère tend à s'éloigner du pignon, phénomène favorisé par la présence de la ligne poussoir. Suivant le sens de rotation du pignon, le système se retrouve alors soit en configuration 1, soit en configuration 2 .

Lorsque le couple revient à zéro, le système revient en configuration 3, ce qui génère des chocs, donc des vibrations.

La propagation des vibrations se fait via les arbres et les roulements vers les carters, puis vers le véhicule par l'intermédiaire des points d'attaches de la direction.

\subsection{Objectifs}

Peu de travaux ont été menés jusqu'à présent sur ce sujet. Les modèles mécaniques mis en place pour l'instant utilisent la théorie du Bond Graph [1-4], et privilégient une seule dimension. Les jeux de denture, les raideurs d'engrènement ainsi que les couplages tridimensionnels sont négligés dans ce type de modélisation. D'autres modèles, cette fois-ci analytiques, existent [5]. Ils sont basés sur les mêmes hypothèses simplificatrices. Un modèle d'ensemble prenant en compte les nonlinéarités physiques du système est développé pour mieux appréhender les phénomènes observés. Ce modèle permet 


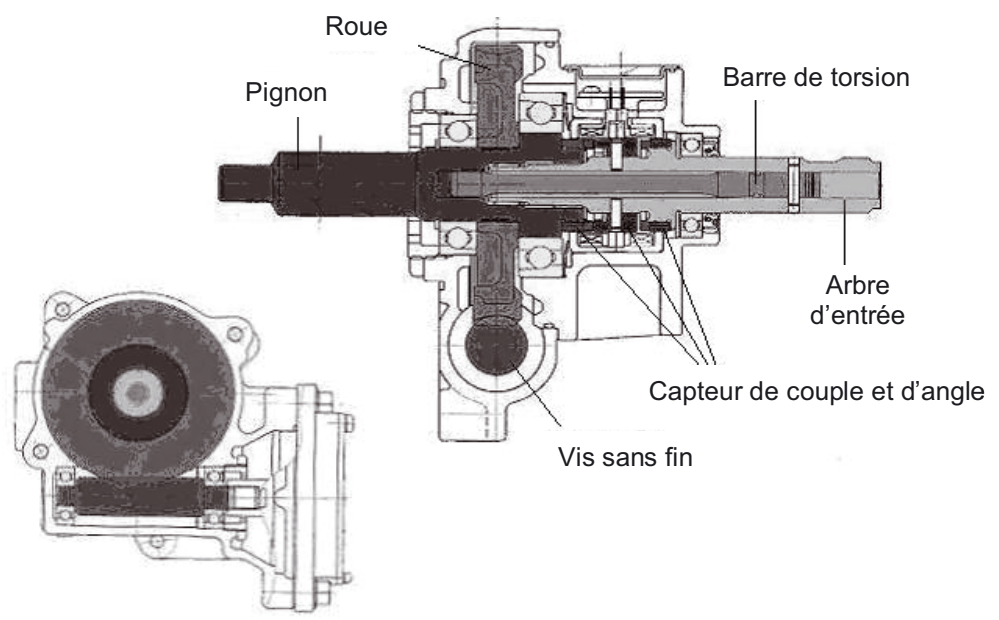

Fig. 4. Module d'assistance.

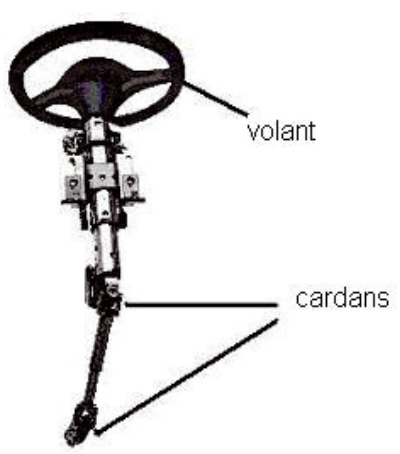

Fig. 5. Colonne de direction.

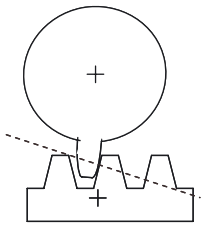

Configuration 1

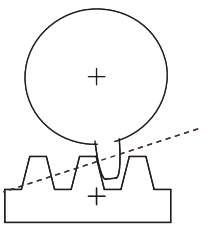

Configuration 2

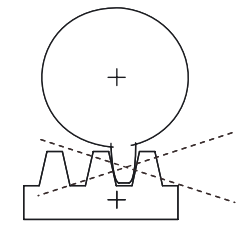

Configuration 3
Fig. 6. Configurations d'engrènement du pignon-crémaillère.

de mieux comprendre le comportement dynamique de la direction, en particulier les modes de transmission des vibrations, et de prévoir le comportement du système.

Les résultats sont présentés avec des échelles adimensionnées, l'abscisse temporelle étant normée sur une période.

\section{Modélisation d'une DAE}

\subsection{Modélisation globale de la direction}

L'ensemble de la DAE est modélisé afin de simuler sa réponse dynamique transitoire lorsqu'elle est soumise à une excitation extérieure. Elle est discrétisée en éléments-finis qui sont assemblés pour former la structure complète. Les déplacements, vitesses et accélérations de chacun des nœuds de la structure sont obtenus par résolution numérique (schéma de Newmark [6]) de son équation du mouvement sous forme matricielle :

$$
[M]\{\ddot{q}\}+[C(\delta)]\{\dot{q}\}+[K(\delta)]\{q\}=\{F(t, \delta)\}
$$

La DAE comporte de nombreuses non-linéarités, tels que les engrenages. Les matrices de raideur et d'amortissement sont donc variables au cours du mouvement : elles dépendent de la configuration de chaque élément non linéaire. Il en est de même pour le vecteur des efforts extérieurs qui dépend, de plus, de l'excitation imposée au système.

\subsection{Les modèles développés}

L'ensemble des modèles développés est basé sur une description géométrique des éléments de la DAE. L'hypothèse de petits déplacements est retenue. Ils sont décrits par Bordegaray [6]. Ils peuvent se différencier entre :

- les éléments linéaires : poutres [7], disques, éléments de masse, de raideur et d'amortissement supplémentaires ;

- les éléments non-linéaires : engrenage roue et vis sans fin [8], engrenage pignon et crémaillère, ressort ABLS, ligne poussoir, prise tournevis, joint de cardans et roulements [9-12].

L'ensemble des modèles développés est validé par des tests en dynamique sur la DAE. Une nouvelle modélisation de la ligne poussoir est présentée ici. 


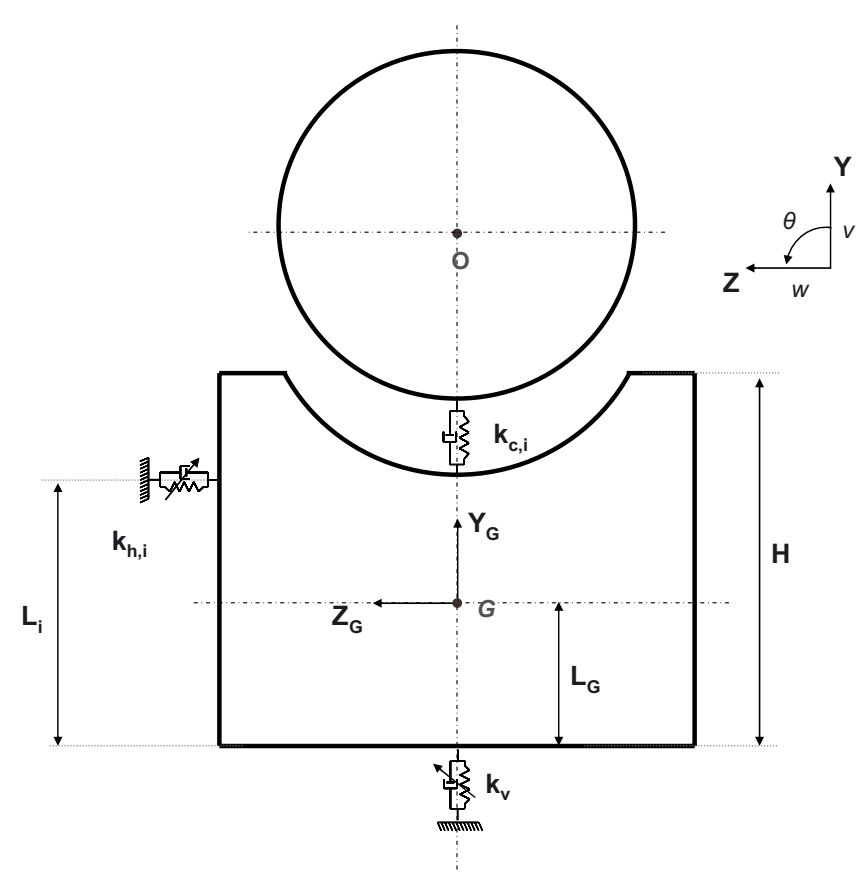

Fig. 7. Modélisation de la ligne poussoir.

\section{Modélisation de la ligne poussoir}

\subsection{Description générale et hypothèses}

Le modèle développé, voir la figure 7 , est un modèle non-linéaire à paramètres concentrés. Le support poussoir est supposé indéformable sauf aux contacts avec la crémaillère de la DAE et de géométrie parfaite. Les liaisons du support poussoir avec le carter de la DAE sont modélisées par des raideurs linéaires par morceaux étant donnée la présence de jeux de fonctionnement. Ce modèle est plan et intégré dans le modèle global tridimensionnel de la chaîne cinématique des DAE.

\subsection{Gestion du contact et angles de contact}

\subsubsection{Fonction jeu}

Les raideurs $k_{\mathrm{c}, \mathrm{i}}$ définies aux contacts possibles du poussoir et de la crémaillère interviennent dans la modélisation du système seulement si le contact est géométriquement possible; soit si l'écrasement au contact considéré est positif ou nul :

$$
\Delta_{i}\left\{\begin{array}{l}
\geqslant 0, \text { contact } \\
<0, \text { pas contact }
\end{array}\right.
$$

On définit ainsi deux fonctions jeu, figure 8, qui permettent de déterminer les raideurs de contact au cours du mouvement du système à partir de $k_{\mathrm{c}, i}$, raideur théorique de chaque contact :

$$
K_{\mathrm{c}, i}=f_{i} k_{\mathrm{c}, i}
$$

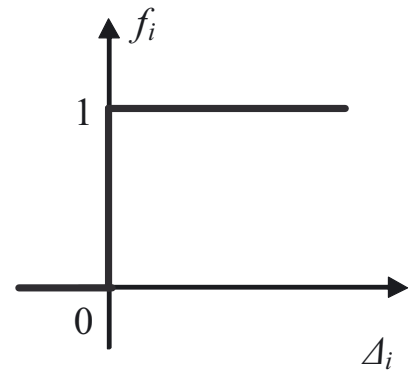

Fig. 8. Fonction de gestion du contact.
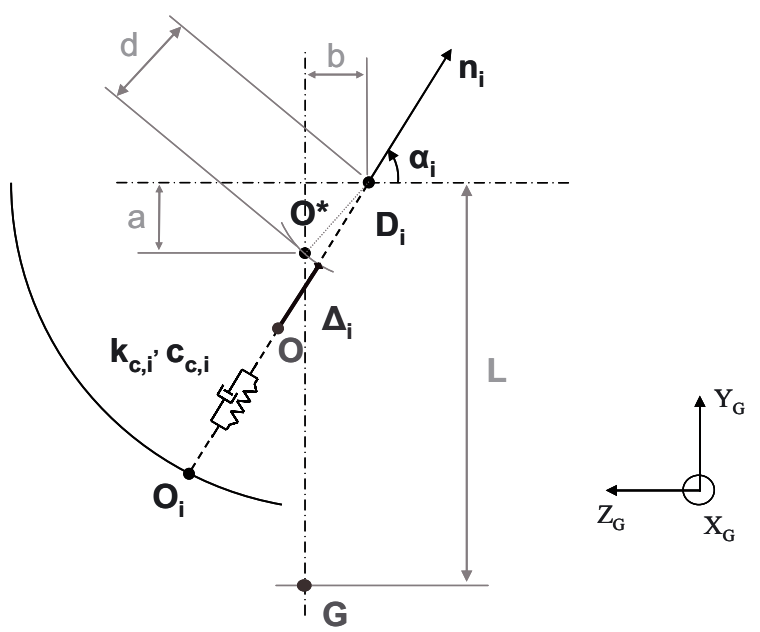

Fig. 9. Écrasement du contact patin/crémaillère.

\subsubsection{Calcul de l'angle de contact}

Les angles de contact $\alpha_{i}$ associés à chaque contact entre la crémaillère et le patin de la ligne poussoir sont actualisés à chaque pas de temps du calcul de la réponse temporelle du problème. Il sont définis tels que, voir la figure 9 :

$$
\tan \alpha_{i}=\frac{v_{\mathrm{G}}-v_{\mathrm{O}}+a}{-\left(w_{\mathrm{G}}-w_{\mathrm{O}}+b_{i}+(L-a) \theta_{\mathrm{G}}\right)}
$$

\subsection{Matrices élémentaires}

\subsubsection{Matrice raideur}

Pour chacune des raideurs $k_{i}$ définies du modèle de la ligne poussoir, l'énergie de déformation associée $U_{i}$ peut être exprimée sous la forme :

$$
U_{i}=\frac{1}{2} k_{i}^{*} \Delta_{i}^{2}
$$

avec $\Delta_{i}$ la déformation de la raideur sous une charge $F$, exprimée en fonction des ddl associés au modèle de la ligne poussoir, et $k_{i}^{*}$ la valeur apparente de la raideur non-linéaire en fonction de $\Delta_{i}$, figure 10 . 


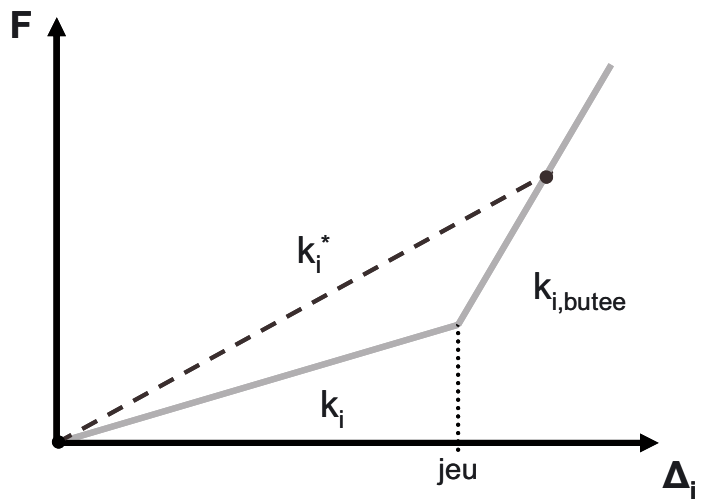

Fig. 10. Force linéaire par morceaux.

Concernant les raideurs axiales et horizontales, les équations de Lagrange permettent de déterminer la matrice de rigidité $\left[K_{\mathrm{p}}\right]$ de la ligne poussoir dans la base des déplacements $q=\left\langle v_{\mathrm{G}} w_{\mathrm{G}} \theta_{\mathrm{G}}\right\rangle$.

$$
\begin{aligned}
{\left[K_{\mathrm{p}}\right] } & =\left[\begin{array}{lll}
k_{11} & 0 & 0 \\
& k_{22} & k_{23} \\
\operatorname{sym} & k_{33}
\end{array}\right] \\
k_{11} & =k_{v} \\
k_{22} & =\sum_{i} k_{\mathrm{h}, i} \\
k_{23} & =\sum_{i}\left(L_{i}-L_{\mathrm{G}}\right) k_{\mathrm{h}, i} \\
k_{33} & =\sum_{i}\left(L_{\mathrm{G}}-L_{i}\right)^{2} k_{\mathrm{h}, i}
\end{aligned}
$$

De la même façon, un effort équivalent de la forme suivante est ajouté au second membre lors du passage d'une raideur $k$ à une raideur $k_{\text {butee }}$ :

$$
F_{\mathrm{p}}=\left(k_{\text {butee }}-k\right) \cdot j e u
$$

Concernant les raideurs de contact, il s'agit d'exprimer les écrasements aux contacts. Les normales aux contacts s'écrivent :

$$
\vec{n}_{i}=\left\{\begin{array}{l}
s_{\alpha_{i}} \\
-c_{\alpha_{i}}
\end{array}\right\}_{R_{\mathrm{G}}}=\left\{\begin{array}{l}
s_{\alpha_{i}}+\theta_{\mathrm{G}} c_{\alpha_{i}} \\
-c_{\alpha_{i}}+\theta_{\mathrm{G}} s_{\alpha_{i}}
\end{array}\right\}_{R_{0}}
$$

Les écrasements s'expriment, voir la figure 9 :

$$
\Delta_{i}=\overrightarrow{O D}_{i} \cdot \vec{n}_{i}-d
$$

En négligeant les termes du second ordre, on obtient :

$$
\begin{aligned}
\Delta_{i} \cong\left(v_{\mathrm{G}}\right. & \left.-v_{\mathrm{O}}+a\right) s_{\alpha_{i}} \\
& +\left(-w_{\mathrm{G}}+w_{\mathrm{O}}-b_{i}-(L-a) \theta_{\mathrm{G}}\right) c_{\alpha_{i}}-d
\end{aligned}
$$

Considérant les équations (3), (5) et (10), les équations de Lagrange permettent de déterminer la matrice du contact poussoir-crémaillère $\left[K_{\mathrm{c}}\right]$ et un vecteur géométrique $\left\{F_{\mathrm{c}}\right\}$ dû aux conditions de contact ajouté au second membre avec les efforts extérieurs.

Ainsi, la matrice de rigidité élémentaire associée à la modélisation de la ligne poussoir est telle que :

$$
[K]=\left[K_{\mathrm{p}}\right]+\left[K_{\mathrm{c}}\right]
$$

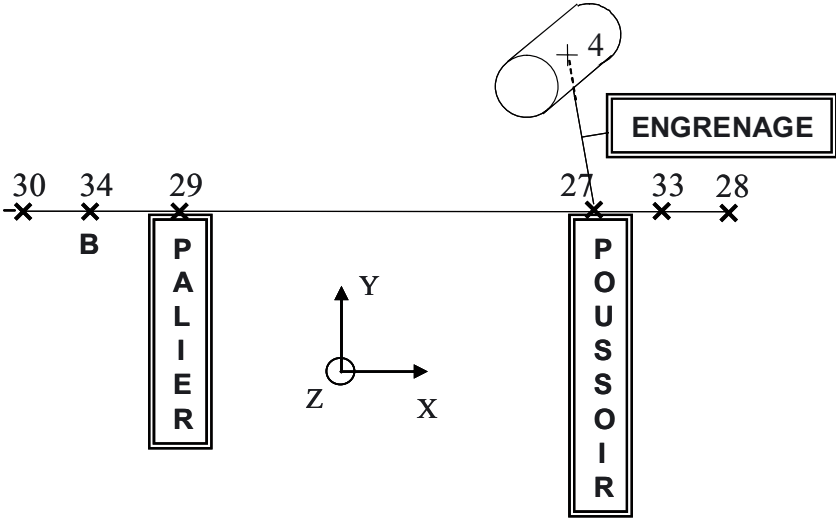

Fig. 11. Modélisation d'une direction manuelle.

\subsubsection{Matrice d'amortissement}

La matrice d'amortissement élémentaire associée à la modélisation de la ligne poussoir est déduite de la même façon des énergies de dissipation de chaque élément d'amortissement (placés en parallèle de chaque raideur) qui s'écrivent :

$$
R_{i}=c_{i}^{*} \frac{\mathrm{d}}{\mathrm{d} t}\left(\Delta_{i}\right)
$$

\subsubsection{Matrice masse}

L'énergie cinétique du système s'écrit :

$$
T=\frac{1}{2} m\left(\dot{v}_{\mathrm{G}}^{2}+\dot{w}_{\mathrm{G}}^{2}\right)+\frac{1}{2} I \dot{\theta}_{\mathrm{G}}
$$

Les équations de Lagrange permettent alors de déterminer la matrice de masse de la ligne poussoir dans la base des accélérations $\ddot{q}=\left\langle\ddot{v}_{\mathrm{G}} \ddot{w}_{\mathrm{G}} \ddot{\theta}_{\mathrm{G}}\right\rangle$ :

$$
[M]=\left[\begin{array}{lll}
m & 0 & 0 \\
& m & 0 \\
\operatorname{sym} & & I
\end{array}\right]
$$

\subsection{Intégration dans un modèle global de direction manuelle}

Le modèle de la ligne poussoir proposé est intégré à la modélisation tridimensionnelle d'une DM, figure 11. Il s'agit d'un assemblage d'éléments poutre pour la crémaillère, d'un élément disque pour le pignon, de l'élément d'interface d'engrènement de l'engrenage pignon et crémaillère, d'un élément ligne poussoir et d'un palier.

\section{Caractérisation et validation}

\subsection{Caractérisation expérimentale de la ligne poussoir}

Les raideurs et les jeux nécessaires à la modélisation de la ligne poussoir et du palier sont caractérisés 

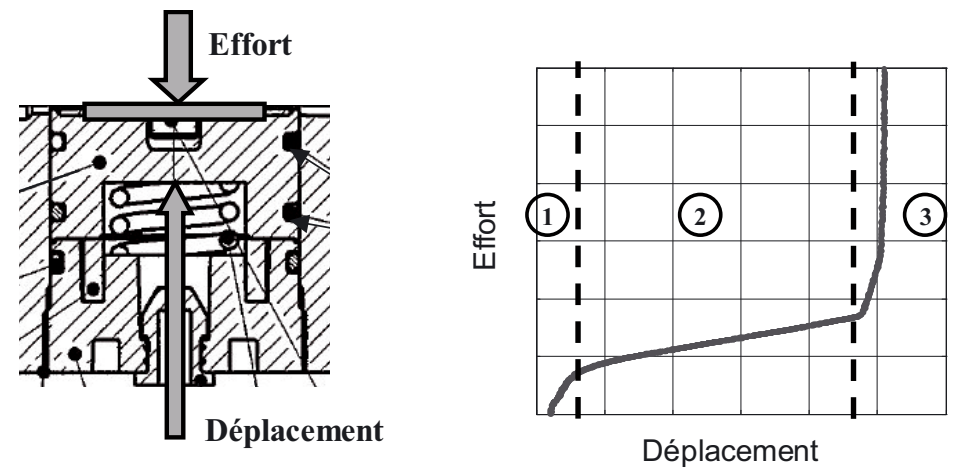

Fig. 12. Compression de la ligne poussoir.
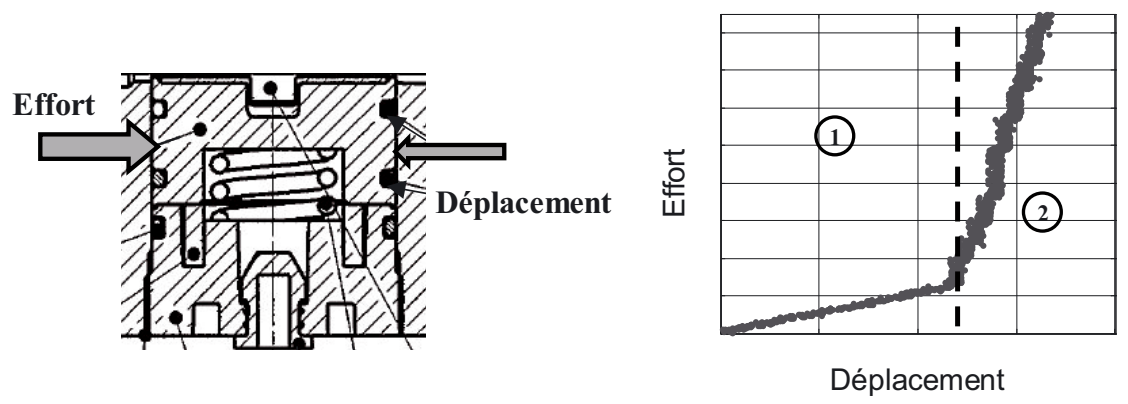

Fig. 13. Effort radial sur la ligne poussoir.
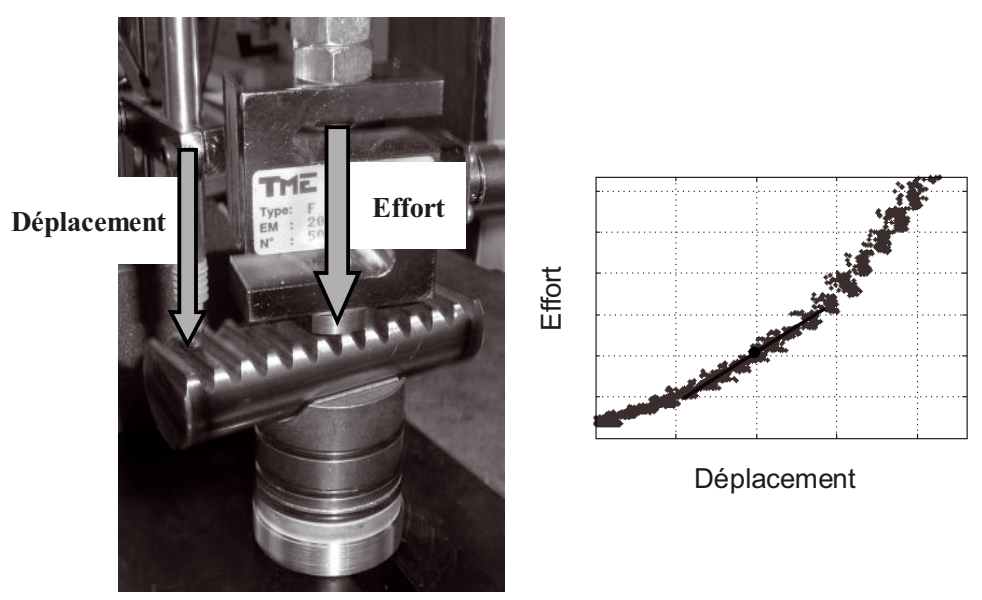

Fig. 14. Effort de contact crémaillère-patin poussoir.

expérimentalement. Les relations effort-déplacement utiles sont déterminées :

- compression de la ligne poussoir, figure 12 : décollement des joints toriques (1), compression du ressort (2) puis compression de l'élastomère et arrivée en butée sur la vis poussoir (3);

- raideur radiale totale de la ligne poussoir, figure 13 : compression des joints toriques (1) puis arrivée en butée sur le carter (2);

- contact crémaillère-patin poussoir, figure 14 : la raideur tangente est déterminée à la précharge de la ligne poussoir ;
- palier, figure 15 : la relation est linéaire pour les amplitudes de déplacements simulés.

\subsection{Validation dynamique du modèle}

\subsubsection{Banc d'essai}

La DAE est montée sur un banc d'essai, figure 16. Les essais consistent à entraîner en rotation l'arbre d'entrée de la direction complète afin d'exciter le système (excitations dues aux non-linéarités). Pour cela, la direction est reliée 

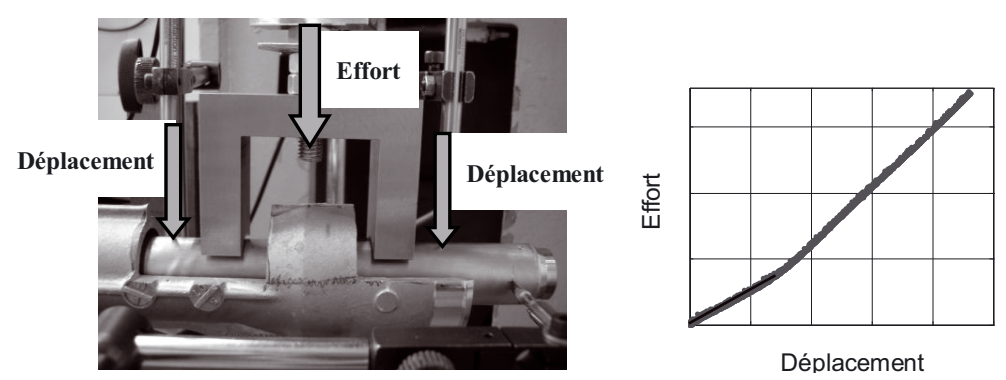

Fig. 15. Effort de compression du palier.

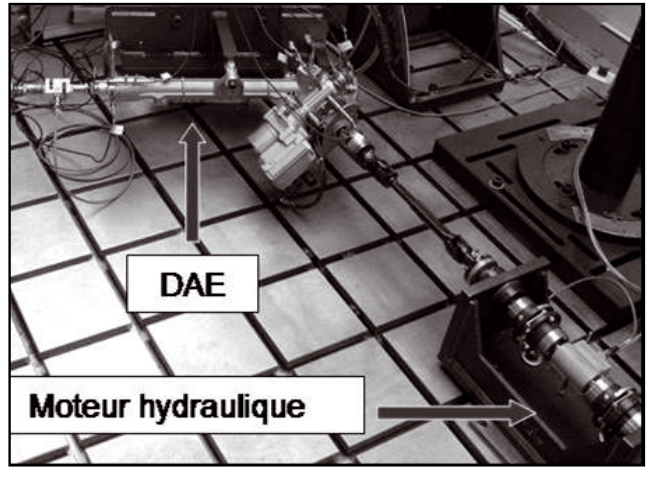

Fig. 16. Banc d'essai dynamique.

par son arbre d'entrée à un moteur hydraulique asservi en rotation.

Une inertie est placée sur la vis de l'engrenage roue et vis sans fin afin de charger le système. Les déplacements de la crémaillère et du poussoir sont mesurés au cours de l'essai, ainsi que la rotation du pignon. Le signal de rotation de l'arbre d'entrée du système de direction sert de donnée d'entrée pour la modélisation de ce banc d'essai.

\subsubsection{Modélisation du banc d'essai}

Le banc d'essai dynamique est modélisé par assemblage des modèles de DM (cf. Sect. 3.4) et du module d'assistance [6]. La modélisation est adaptée à la configuration de mise en charge du banc.

\subsubsection{Comparaison des résultats expérimentaux et de simulation}

La rotation de l'arbre d'entrée, figure 17, mesurée sur le banc expérimental est imposée sur l'arbre d'entrée de la DAE modélisée.

Il s'agit de comparer les mouvements expérimentaux et de simulation des éléments de la chaîne cinématique de la DAE, en se focalisant sur ceux de la ligne poussoir elle-même ainsi que des éléments dont le comportement est directement influencé par la ligne poussoir.

La rotation du pignon, figure 18, simulée se superpose avec la rotation mesurée sur le banc expérimental. Il en est de même pour la rotation de la vis, figure 19, et la
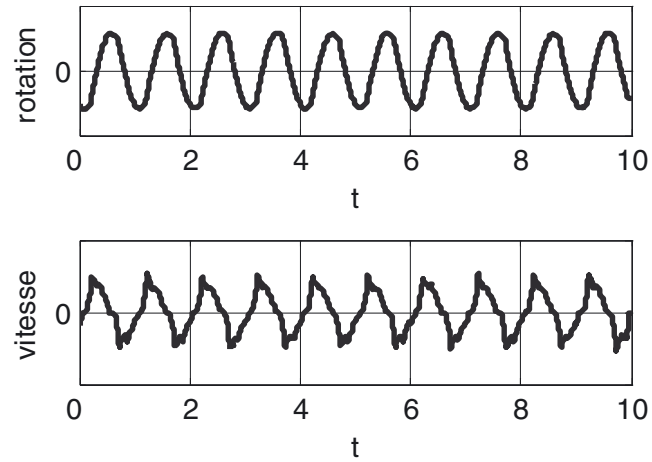

Fig. 17. Mouvement imposé sur l'arbre d'entrée.

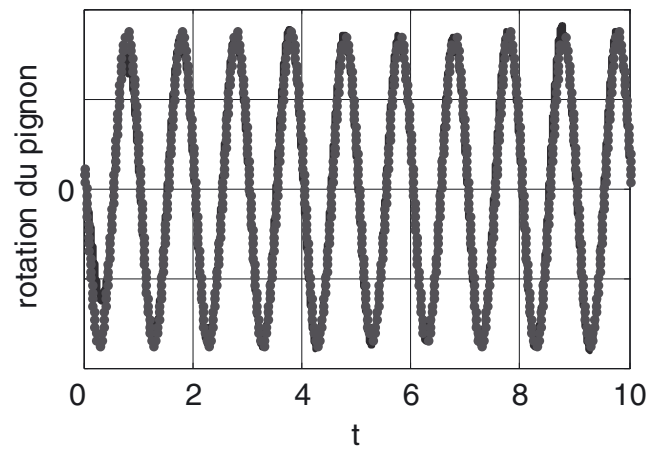

Fig. 18. Rotation du pignon mesurée (...) et simulée (--).

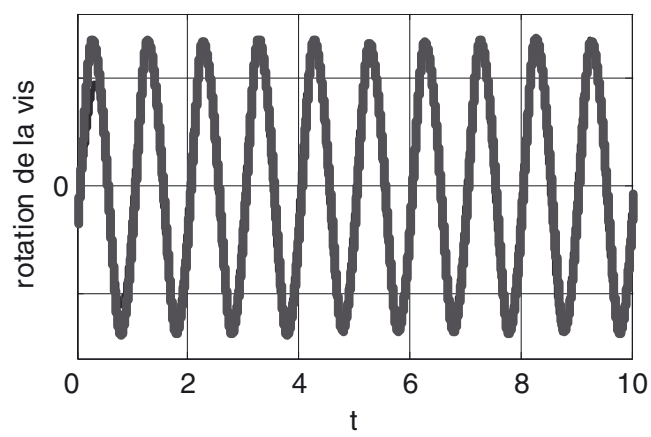

Fig. 19. Rotation de la vis mesurée (...) et simulée (--).

translation de la crémaillère, figure 20. La barre de torsion du système, les frottements présents dans le système ainsi que les engrenages influencent principalement ces mouvements. Leur modélisation a été validée [6]. 


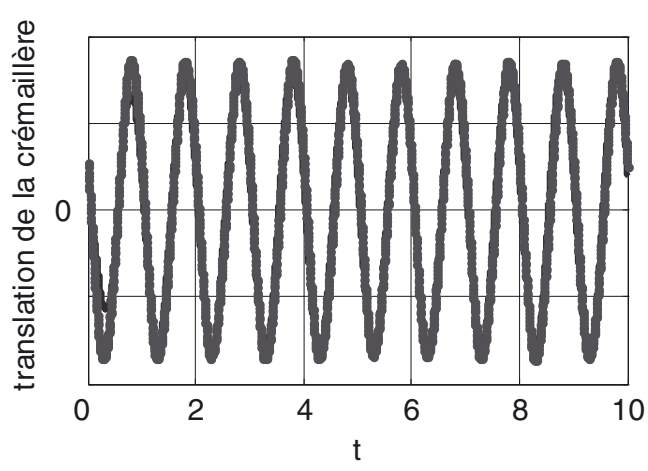

Fig. 20. Translation de la crémaillère mesurée (...) et simulée $(--)$.

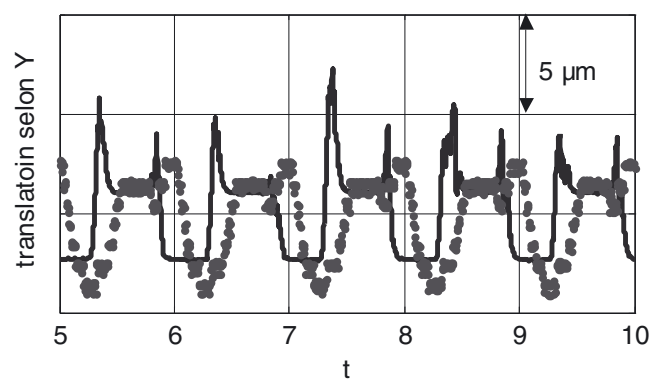

Fig. 21. Translation axiale du poussoir mesurée (...) et simulée (--).

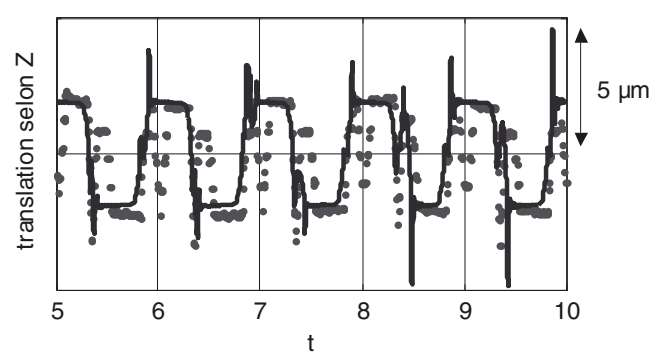

Fig. 22. Translation radiale du poussoir mesurée (...) et simulée (--).

Le déplacement du poussoir est mesuré dans le plan de modélisation, figure 11 : dans son axe (recul poussoir, axe $Y$, Fig. 21) et dans la direction perpendiculaire (axe $Z$, Fig. 22). Ces mouvements, d'ordre de grandeur très faible, sont correctement simulés, en amplitude et en fréquence. La bonne reproduction de ces mouvements est importante pour simuler correctement le comportement radial de la crémaillère : en effet, ce sont les mouvements $\mathrm{du}$ poussoir, en particulier sa rotation, figure 23 , qui vont permettre les mouvements radiaux de la crémaillère.

Les mouvements radiaux de la crémaillère au point $\mathrm{B}$ (nœud 34, Fig. 11) sont mesurés dans le plan normal à la crémaillère : selon les axes $Y$ et $Z$.

Le modèle proposé pour la ligne poussoir permet de simuler le bon comportement radial pour la crémaillère. Dissocier le poussoir de la crémaillère, et prendre en compte les mouvements de basculement possibles de celuici, ainsi que l'angle de contact variable entre celui-ci et la crémaillère ont permis d'arriver à ce résultat.

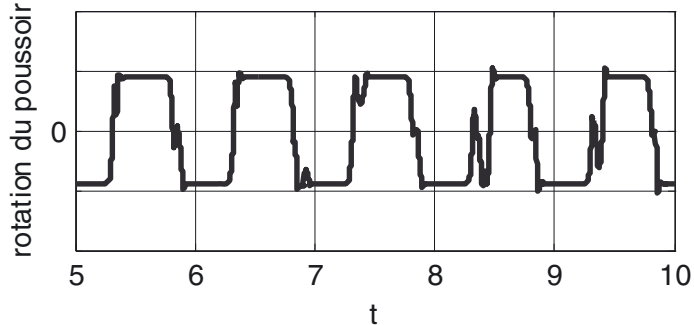

Fig. 23. Translation simulée du poussoir.
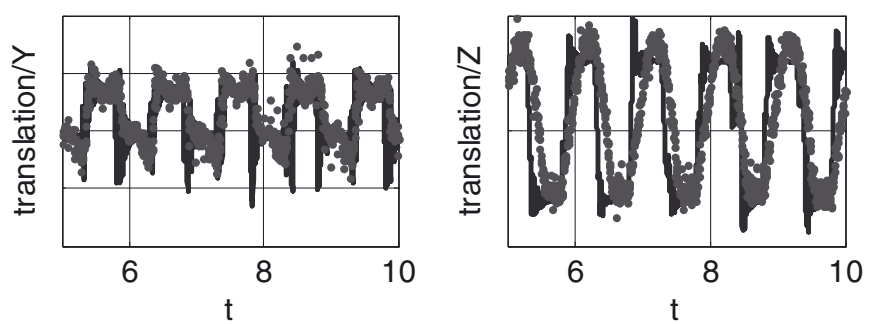

Fig. 24. Translation du point B de la crémaillère mesurée (...) et simulée (--).

\section{Conclusions}

Un modèle de la ligne poussoir des DAE est développé et caractérisé. La comparaison des résultats de simulation et expérimentaux montre que le modèle proposé permet de reproduire le comportement en amplitude et en fréquence des différents éléments de la DAE observés, en particulier ceux de la crémaillère. Le modèle dynamique développé est donc validé et pourra être utilisé dans les phases de conception du système. Une étude des chocs dans la DAE basée sur l'ensemble des modèles évoqués sera menée. La modélisation de la chaîne cinématique de la DAE sera également étendue jusqu'aux interfaces avec le véhicule afin d'étudier l'impact de la DAE sur son environnement.

Remerciements. Les auteurs remercient JTEKT Europe pour son soutien technique et financier.

\section{Références}

[1] S.M. Taheri, S. Karim, M.G. Mohamed, Dynamic analysis of a hydraulically assisted rack and pinion power steering system using bond graph, in: ASME Conference on ESDA, London, England, 1994

[2] Q. Zhang, D. Wu, J.F. Reid, E.R. Benson, Model recognition and validation for an off-road vehicle electrohydraulic steering controller, Mechatronics 12 (2002) 845-858

[3] P. Simon, Numerical Simulation of Electric Power Steering (EPS) System, Koyo Engineering J. English Edition 161 (2002) 52-56

[4] A. Badawy, J. Zuraski, F. Bolourchi, A. Chandy, Modeling and analysis of an electric power steering system, SAE Technical Paper Series 1999-01-0399, 1999 
[5] A.B. Proca, A. Keyhani, Identification of power steering system dynamic models, Mechatronics 8 (1998) 255-270

[6] C. Bordegaray, Comportement dynamique des directions assistées électriques : théorie et expérimentations, Thèse, ISAL007, 2007

[7] P. Couderc, Comportement dynamique des chaînes de transmission automobiles, Thèse, ISAL0063, 1997

[8] C. Bordegaray, G. Ferraris, M. Guingand, J.P. de Vaujany, Dynamic behavior of a worm gear, in: Proceedings of DETC'05, ASME 2005 Design Engineering Technical Conferences and Computers and Information in Engineering Conference, Long Beach, California USA, 2005
[9] F. Besson, G. Ferraris, M. Guingand, J.P. de Vaujany, Simulation of the dynamic behavior of Electric Power Steering systems using dedicated finite elements, JSDD 3-3 (2009) 429-440

[10] T.C. Lim, R. Singh, Vibration transmission through rolling element bearings, part I: Bearing stiffness formulation. J. Sound Vib. 139-2 (1990) 179-199

[11] T.C. Lim, R. Singh, Vibration transmission through rolling element bearings, part II: system studies. J. Sound Vib. 139-2 (1990) 201-225

[12] F. Lahmar, Interactions entre la dynamique de l'engrènement et les paliers à roulements, Thèse, ISAL0020, 2000 